\title{
Effects of Socioeconomic diversity on iPod Touch Device Use in Real-World Environments
}

\author{
Chad C. Tossell $^{1}$, Jo R. Jardina ${ }^{2}$, Philip T. Kortum ${ }^{1}$, S. Camille Peres ${ }^{2}$, Clayton W. Shepard ${ }^{1}$, Ahmad \\ Rahmati $^{1}$, Lin Zhong ${ }^{1}$ \\ ${ }^{1}$ Rice University \\ ${ }^{2}$ University of Houston-Clear Lake
}

The iPod Touch provides portable and personalized information, entertainment, and communication resources for users. The goal of this study was to assess the influence of user diversity on how these handheld mobile computers are employed in real environments. Using an unobtrusive, longitudinal methodology to collect data, we explored how different socioeconomic groups personalize and operate iPod Touches. The lower income group used the instrumented iPod Touch more often and for different purposes. Design implications for newer-generation handheld devices and content are discussed.

\section{INTRODUCTION}

Handheld mobile technologies are becoming ubiquitous. Recent numbers show that over 60 million people own at least one smartphone in the United States (Marcom, 2010). Newer smartphone technologies have changed how information seeking takes place (Matthews, Tang \& Pierce, 2009). In almost any environment, users have fast access to the Web, social networking, location information, gaming, and hundreds of other applications developed specifically for portable technology.

Lower cost alternatives to smartphones, such as the iPod Touch, offer advanced and usable capabilities without recurring service costs. A recent report showed that iPod Touches account for over half of the handheld devices offered by Apple (AdMob, 2010). Over 65\% of those that own iPod Touches are below the age of 17 . iPod Touch use is also robust; iPod Touch owners download 37\% more applications per month than iPhone and Android users (AdMob, 2010). Clearly, this technology is an important vehicle to provide mobile applications and services to a growing number of users.

Despite their widespread use, there is little research on what people actually do on these devices. More naturalistic studies can assess the impact of human factors, such as user diversity, on device usage. Emerging logging methodologies offer this access without self-reports, focus groups, or physical observations. Designers of mobile content and applications could leverage studies on user diversity to provide more customized capabilities to targeted users.
The goal of this study was to assess the influence of socioeconomic diversity on how these handheld mobile computers are employed in real environments. Using an unobtrusive longitudinal methodology to collect data, we explored how differing socioeconomic groups personalized and operated iPod Touches.

\section{Background}

Understanding user diversity is a central tenet of human factors (Shneiderman, 2000). Indeed, much research in human factors is geared toward designing to accommodate users' particular knowledge, skills, age, gender, income, and handicaps among other things (Schneiderman, 1999). The design goal in this regard is universal accessibility and usability for a broad range of users. Interestingly, there is little recent research on designing differently to accommodate users in different socioeconomic strata.

Differences in computing access, driven by factors such as socioeconomic status (SES), could lead to different needs for technological capabilities. Just last year, Pew (2010) reported that over 59\% of Americans are accessing the Web on handheld mobile devices with an increasing trend. A larger number of low income users without access to additional resources were reported as a central driver of this increase.

Unfortunately, the usability of smartphones is poor for many tasks. There are well-documented human factors problems including small screen interfaces, awkward text entry, and slow network speeds when accessing the Web from many of the previous- 
generation smartphones (Nielsen, 2009). Small screen interfaces make it challenging for users to read (Duchnicky \& Kolers, 1983) and enter data (Tossell, Kortum, Shepard, Rahmati \& Zhong, 2010). Additionally, network wait-times are a "bottleneck" for uninterrupted usage and a challenge for enhanced user experiences (Roto \& Oulasvirta, 2005). Designers of smartphone devices and applications have been working to mitigate these limitations through the development of different input techniques and optimized content for small screens respectively.

Newer handheld mobile devices, led by the iPhone and iPod Touch, have reduced many of these concerns. The enhanced usability has led to increased accessibility and is changing the way technology is employed in real environments (Matthews, Pierce \& Tang, 2009). The combination of more powerful mobile devices (the iPod Touch is as powerful as a 1993 computer) and optimized mobile applications and Web content has made retrieving and employing information much more efficient (Tossell et al., 2010).

\section{Approach}

While there is previous research that shows what smartphone owners do on smartphones (e.g., Falaki, Lymberpoulous, Mahajan, Kandula \& Estrin, 2010), no previous work has assessed demographic factors to establish user differences. Further, many of the previous studies are focused on the use of prior-generation mobile phones. The lack of studies assessing what user populations do on newer-generation devices, such as the iPhone and iPod Touch, drives the current study to fill this gap by capturing real-world iPod Touch device usage. Moreover, our goal is to better understand how particular human factors, such as user diversity and access to resources, influence operation of these devices.

The structure of the rest of this paper is as follows. First, we describe the naturalistic method used to collect data. Second, we present how applications are installed on iPod Touches and the types of activities users carry out on these devices that lack the standard communication modalities (e.g., voice phone, SMS). Third, we explore the most frequently accessed applications as well the applications that consumed the greatest amount of time. Finally, we report the effects of SES and additional resources on how the device is used.

\section{METHOD}

An unobtrusive logger was employed to capture all user interactions with iPod Touches. Below we give a brief overview of the method applied without substantial detail of the logging technology. For more details on the technical implementation of the logger, see Shepard, Rahmati, Tossell, Kortum, \& Zhong (2010).

\section{Participants}

iPod Touches were provided to 14 students at the University of Houston-Clear Lake to use over the course of six months. Some of the participants $(n=8)$ already owned smartphones and used the iPod Touches as secondary devices. Participants were assigned to groups based on their SES. Subjects with total annual income below \$40,000 (for the entire household) were put in the Low SES group $(n=7)$ and subjects with total annual income above $\$ 40,000$ (for the entire household) $(n=7)$ were assigned to the High SES group. Everybody in the low SES group also had a family size greater than 3 . In total, the High SES group contained 5 participants that used another smartphone during the study and the Low SES group contained 3 smartphone owners.

\section{Materials}

iPod Touches are multi-faceted devices that provide capabilities to search the Web, send email, play games, and listen to music among other things. Similar to iPhones, applications from Apple's AppStore can be installed and employed. In contrast to iPhones, iPod Touch users do not have ubiquitous access to a data network (e.g., 3G). Thus, in order to get on the Web, a wireless network must be available. The participants received iPod Touch $8 \mathrm{G}$ devices running iOS 3.1.3 for use during the study period.

\section{Procedure}

After signing an informed consent statement, participants were given iPod Touches free of charge to use in conjunction with a statistics class at the University of Houston in Clear Lake. Users operated their devices naturally over the course of six months. No limitations on iPod Touch use were imposed on participants. The logger was designed to capture actual usage data in an unobtrusive way in real environments. Specific data were recorded from each subject over a six-month period. These data included all app names, their associated number of launches and duration of usage. Privacy was maintained by setting constraints on the logger and minimizing researcher-participant interactions. Additionally, subject numbers were assigned to each participant and associated with their data (instead of their names). 


\section{RESULTS}

Figure 1 shows the proportion of accesses for each application as well as the time each application consumed. Users largely spent their time on the device using four applications. Specifically, over $75 \%$ of total duration on the device was spent on the Web, playing games, adjusting the settings, and listening to the iPod Music player. Additionally, these four applications were launched more than others and consumed more than half of total application launches.

On average, subjects installed 17 applications from the AppStore with high variability across users $(S D=$

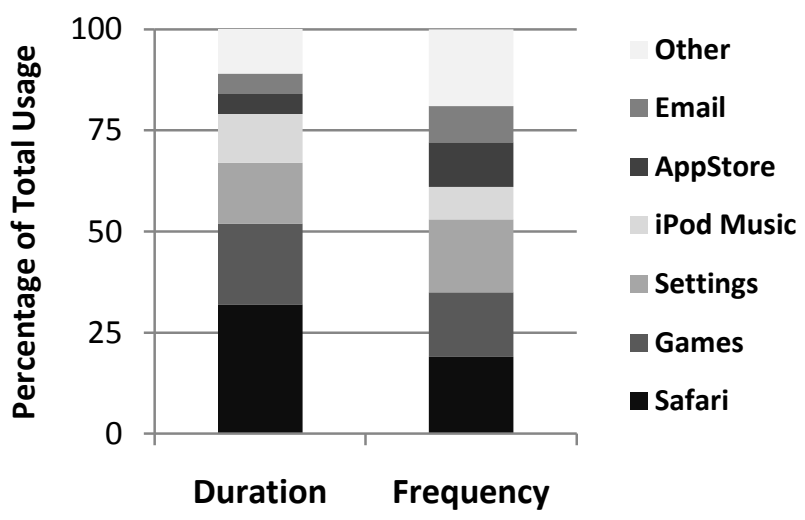

Figure 1. Percentages of time $\&$ number of launches on iPod Touch applications.

27.72). Five users did not install any applications. Four of these five users owned a smartphone. Facebook was the top application downloaded $(n=5)$. However, after manually categorizing all of the applications into several categories, games were installed far more than any other type of application (Figure 2).

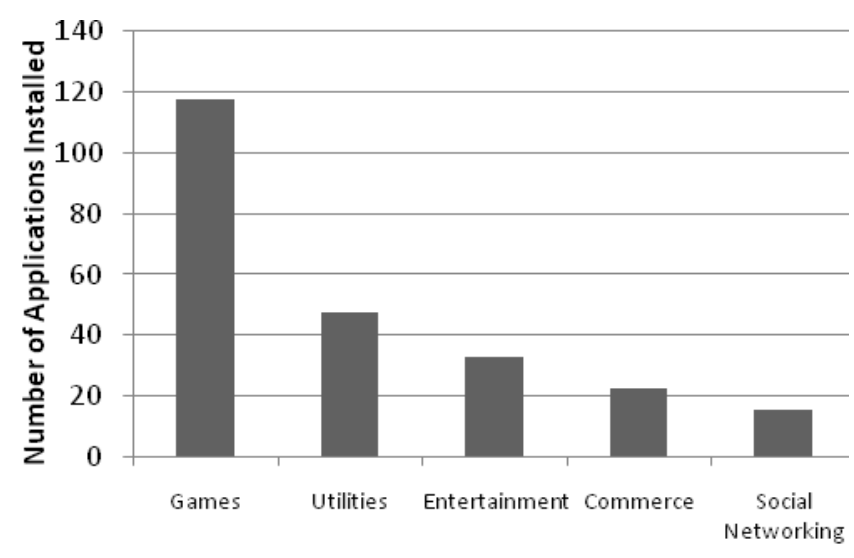

Type of Application

Figure 2. Total number of applications installed by category.
A 2 (SES: Low vs High) x 2 (Device: Smartphone Owner vs Non-Smartphone Owner) factorial analysis of variance (ANOVA) was used to assess differences on the number of applications installed. Low SES users downloaded many more applications $(M=38.6, S D=$ 12.67) compared to their higher SES peers $(M=9.63$, $S D=10.62)$ over the course of the study period $(F(1,10)$ $=5.41, p=.04)$. Additionally, there was a main effect for Device Ownership $(F(1,10)=6.29, p=.03)$. The interaction was slightly above the significance level of $.05(F(1,10)=4.87, p=.06)$; so, this provides some evidence that smartphone owners access the iPod Touch less and this is especially prevalent in higher SES brackets (Figure 3).

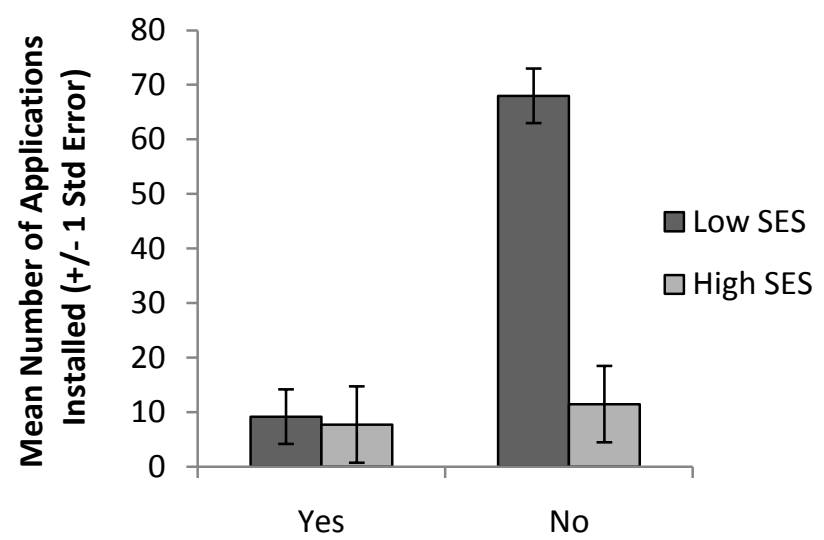

Smartphone Ownership

Figure 3. Mean number of applications installed as a function of smartphone ownership and SES.

A similar ANOVA was used to compare device usage. Frequency of application launches were compared first. A main effect was found for both SES $(F(1,10)=8.53, p=.02)$ and Device $(F(1,10)=5.40, p$ $=.045)$. The low SES group and non-smartphone owners accessed their iPods more than the other groups and no interaction was found $(F(1,10)=2.73, p=.13)$.

Specific application usage was also evaluated between these groups to examine what drove these usage differences. Interestingly, no real differences existed in the number of gaming applications launched $(F(1,10)=$ $2.10, p=.48$ ). However, a significant interaction (Figure 4) revealed the non-smartphone owners in the Low SES group used Safari more frequently $(F(1,10)=5.17, p=$ $.04)$. The Settings application was also accessed more by the lower SES group $(F(1,10)=7.53, p=.02)$; however, the number of accesses to both Settings and Safari were not correlated $(r=.38, p=.28)$.

Duration on the device was also used as a dependent variable to assess differences between users. 


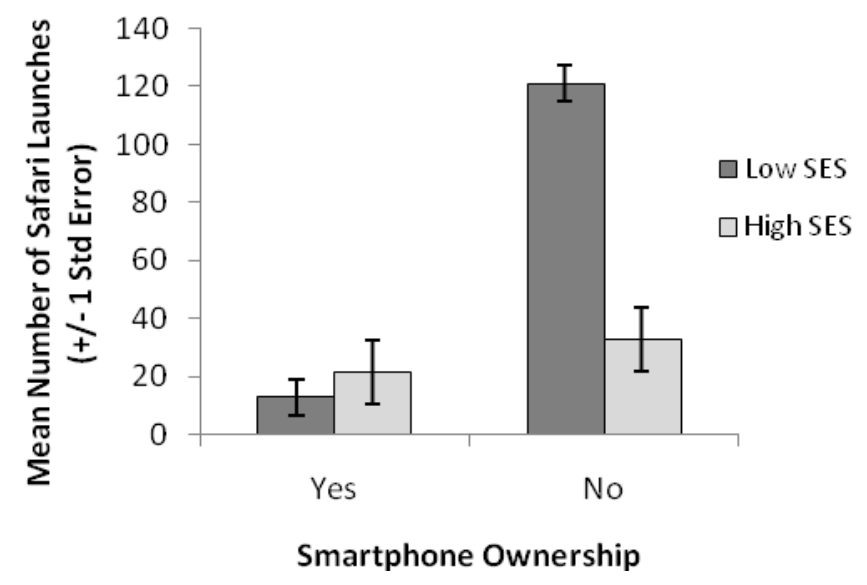

Figure 4. Number of Web accesses as a function of SES and smartphone ownership.

Statistical tests similar to above yielded no effect of SES or smartphone ownership on total usage or top applications usage.

\section{DISCUSSION}

The results reveal several interesting facets regarding iPod Touch use and the digital divide. Even with a small sample size, several findings are noted.

First, some of the most interesting results were the applications not used by our participants. News, Timer, Calendar, and even Facebook made up a small percentage $(<5 \%)$ of overall device usage. In fact, we were surprised with how little these devices were used overall. It seems that the primary driver of this result was due to access to another smartphone and lower SES levels as seen in Figures 3 and 4. Of course, at a broader level, a lack of personal investment in the product and wireless access could have influenced the low amount of use as well.

When these devices were used, participants spent a majority of their time using the Web, playing games, and listening to music. These functions are similar to some traditional desktop computing tasks. Although iPhones, with full communication capabilities (i.e., phone \& texting), seem to complement traditional fixed-based computing tasks, iPod Touches seemed to be used for tasks that replicate those kinds of tasks performed on less-portable computers (e.g., laptops, PCs). This is consistent with the hypothesis that certain groups are using the iPod Touch to supplant tasks that would be accomplished on a larger computer, rather than a mobile computer supplement. In this sense, the iPod Touch is more like a handheld mobile computer than a smartphone.

Why was such a large amount of time spent in the Settings application relative to other applications across users? We speculate that because iPods do not have $3 \mathrm{G}$ network access, participants had to access wireless networks (using the settings application in order to use the device). The lack of a ubiquitous network (e.g., 3G) could have produced these additional user interactions with the device.

User socioeconomic diversity played a role in how the iPod Touches were operated. Low SES users seemed to have a greater reliance on the device. This manifested in the increased number of accesses compared to their higher SES peers. However, the amount of time spent on the device was similar. This suggests that while users initiate device accesses based on needs in particular contexts, the amount of time they spend on each application is determined more by situational constraints.

The iPod Touch offers different capabilities compared to smartphones. Indeed, one participant mentioned that she uses her iPod Touch instead of her smartphone to avoid interruptions. This functionality without traditional communication modalities may be desired by certain groups. There is some evidence that parents have already figured this out. As noted earlier, the highest demographic using iPod Touches are youth under the age of seventeen. Although somewhat counterintuitive, it seems clear that network connectivity without synchronous communication capabilities (i.e., telephone and texting) is desirable for some user groups.

\section{Design Implications}

Clearly, not all handheld mobile device users are the same and we have shown here that differences in usage can be driven by demographic factors. Low SES users who did not own a smartphone had different usage patterns compared to higher SES users. Recall from figure 4 that their Web session counts were nearly seven times higher than other users. We believe that this may be because these users are more reliant on the device than peers who own a smartphone or are in a higher SES bracket and may have access to these services in other ways. This suggests that, for the low SES group without an additional smartphone, the iPod does not have special interface characteristics that are driving these results, but rather that the iPod is relied on more for necessary computing tasks. It seems that smartphone ownership, or potentially access to other computer resources, would attenuate the digital divide at some level. Thus, the 
design of lower cost devices with similar capabilities might be beneficial.

The lower SES group also downloaded over four times more applications than their higher SES peers, a substantial difference. This is also likely driven by their lack of access to resources and their increased dependency on the iPod Touch. This suggests that there are clear opportunities to design applications to assist these users in various environments. As demonstrated in Figure 2, the majority of these downloaded applications do not depend on wireless connectivity in order to function. Thus, the lack of a ubiquitous network seemed to be driving lower SES users to install more stand-alone applications that did not require wireless connectivity. Application designers need to consider implementations that do not rely on the Web or cloud to exploit iPod Touch usage. This is particularly true if targeted users are in lower SES brackets.

On the hardware side, wireless access without a ubiquitous network remains a challenge. Low income users relied on their iPod Touches for Web access more than others in this study. Offering more network access in both personal and professional settings would certainly seem to benefit this group. Even in places where wireless was available, it seemed that many had difficulties accessing the wireless network. While it is hard to tell with our data why users were in the Setting application so often, this is one reasonable conclusion. If this conjecture is at least partially accurate, hardware engineers need to consider cleaner and more efficient connections to wireless access points.

This research has shown that there are important factors that impact the use of iPod Touches. One important message for designers is that they need to have a greater understanding of user diversity in order to design effective, efficient and targeted mobile services and devices.

\section{ACKNOWLEDGEMENTS}

This work was supported in part by National Science Foundation (NSF) Awards IIS/HCC 0803556, DUE 9751307, DUE 0089435, and DUE 0919818.

\section{REFERENCES}

AdMob Mobile Metrics Report (2010). Retrieved from: http://metrics.admob.com/wpcontent/uploads/2010/02/AdMob-MobileMetrics-Jan-10.pdf

Duchnicky, R.L. \& Kolers, P.A. (1983). Readability of text scrolled on visual display terminals as a function of window size. Human Factors, 6, 683 $-692$.

Falaki et al. (2010). Diversity in Smartphone Usage. $8^{\text {th }}$ International Conference on Mobile Systems.

Marcom Professionals (2010). Retrieved from: http://www.marcomprofessional.com/posts/david. deans/60.7-million-people-in-the-u.s.-ownsmartphones

Matthews, T., Pierce, J. \& Tang, J. (2009). No Smartphone is an Island. IBM Technical Report RJ10452.

Nielsen, J. (2009). Mobile Usability. Retrieved from: http://www.useit.com/alertbox/mobileusability.html

Pew Internet \& American Life Project (2010). Retrieved from: http://www.pewinternet.org

Roto, V. \& Oulasvirta, A. (2005). Need for non-visual feedback with long response time in Mobile HCI. In Proc. of www2005, May 2005.

Shepard, C., Rahmati, A., Tossell, C., Kortum, P. \& Zhong, L. (2010). LiveLab. Measuring smartphone owners in the field. Presented at HotMetrics 2010. N, NY.

Shneidermann, B. (1999). Universal Usability: Pushing HCI Research to Empower Every Citizen. HCIL Technical Report No. 99-17. University of Maryland.

Shneidermann, B. (2000). Universal usability, Communications of the ACM, 43, 85-91.

Tossell, C., Kortum, P., Shepard, C., Rahmati, A. \& Zhong, L. (2010). Assessing the Performance of Common Tasks on Handheld Mobile Computers. Proc. of Human Factors \& Ergonomics Society $50^{\text {th }}$ Annual Meeting. San Francisco, CA. 